\section{Cardiovascular responses to auditory stimuli}

F. BARRY KEEFE, Navy Medical Neuropsychiatric Research Unit, San Diego, Calif. 92152, and L. C. JOHNSON, San Diego State College, San Diego, Calif. 92115

Three cardiovascular responses to a moderate tone (about $70 \mathrm{~dB}$ ) were studied in 25 Ss. Finger-pulse (FP) response was monophasic vasoconstriction. Head-pulse ( $H P$ ) response was biphasic; vasoconstriction preceded vasodilation. Heart-rate (HR) response usually consisted of an initial small deceleration followed by $a$ more marked acceleration and $a$ secondary deceleration. HP constriction and HR acceleration occurred almost simultaneously; their response magnitudes were highly positively correlated. FP and $H P$ constriction responses were also positively correlated. All responses showed some effect of their prestimulus level.

This report discusses three cardiovascular responses to auditory stimuli: finger pulse (FP), forehead pulse (HP), and heart rate (HR). The shape and magnitude of each variable, the effect of prestimulus level, and the interrelations between the variables will be discussed

\section{METHOD}

Twenty-five young adult Ss ( 24 male, 1 female) were presented $141,000-\mathrm{Hz}$ tones of 0.5 -sec duration with ISI of 30 or $45 \mathrm{sec}$ through dual speakers. Tone intensity was about 40 dB above auditory threshold-measured at head level at 65 to $75 \mathrm{~dB}$ referenced to .0002 dynes $/ \mathrm{cm}^{2}$. The 10-min stimulus period was preceded and followed by $10 \mathrm{~min}$ of no stimulus. Ss were seated with their eyes closed in an air-conditioned, semisoundproof room with instructions to stay awake and listen for the tones. Finger- and forehead-pulse amplitudes were obtained from two photocrystal reflecting plethysmographs (time constant, $.3 \mathrm{sec}$ ) attached to the left thumb and to the center of the forehead respectively. Heart rate was obtained with an Offner cardiotachometer from chest electrodes.

Finger- and forehead-pulse amplitudes were measured in millimeters, heart rate was measured in beats per minute. Each response was measured for 3 pre- and 25 poststimulus pulses or beats. Two methods were used to describe each response. First,

Fig. 1. Right, average pulse-by-pulse response curves computed over $\mathrm{Ss}$ at Trials 1,2 , and 14; left, response curves using points of mean maximum change over Ss.

MEAN MAXIMUM CHANGE

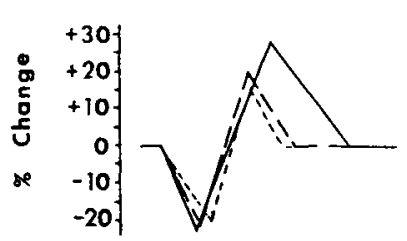

pulse amplitude and HR for each pre- and poststimulus pulse were averaged over $S s$ at each trial (Fig. 1, right). This differs from the usual average evoked response in that the averaging is over $\mathrm{Ss}$ for a given trial and not over trials. Second, the mean maximum change(s) from prestimulus level was found and plotted on the mean poststimulus pulse (beat) at which the maximum change occurred to produce the response diagrams of Fig. 1, left. The mean maximum changes for finger and forehead pulse are expressed in percent change from prestimulus level. The measure of the maximum percent change for $F P$ and $H P$ constriction was the difference between the prestimulus pulse amplitude and the smallest poststimulus pulse amplitude, divided by the prestimulus pulse amplitude. For HP dilation, the maximum percent change was the difference between the prestimulus pulse amplitude and the largest poststimulus pulse amplitude following the initial constriction, divided by the prestimulus pulse amplitude. The HR response was divided into three components and each was scored as beats per minute change from the prestimulus level: (1) early deceleration = lowest HR of Poststimulus Beats 1-3, (2) acceleration =

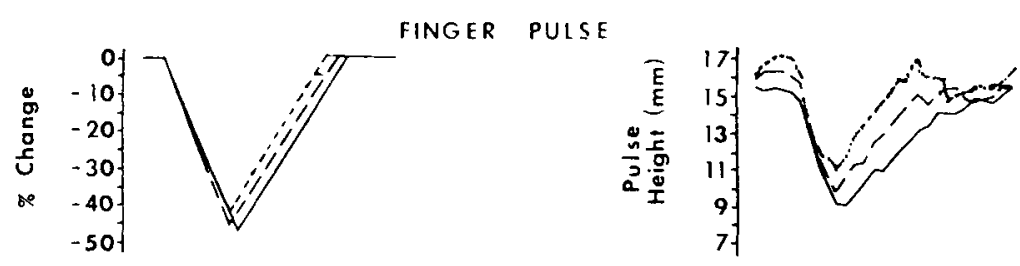

HEAD PULSE

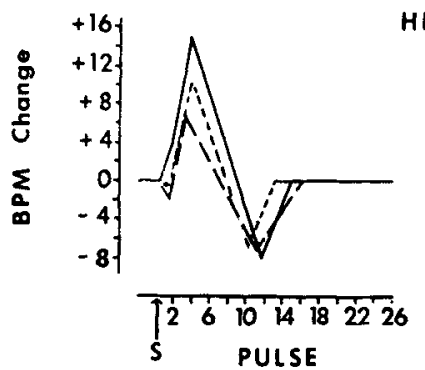

HEART RATE

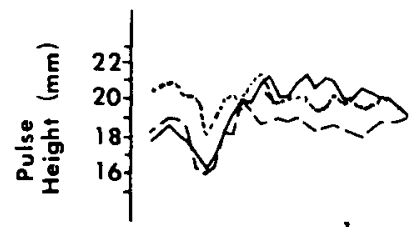

nce between
neceleration rates watiow, 1962).
compared between
with the t test for
LTS
Shape
response was a
triction. The mean
occurred at about
pulse, and its
oximately a $45 \%$
the timing of this
sistent over Ss, the
mplitude is clearly
se-by-pulse average
re no significant
FP responses for
response was
mean maximum
lse
occurred at about
was followed by
of $15 \%$ to $28 \%$ at
pulse. As with the
timing of the
s clearly evident in
average curves. In acceleration within Poststimulus Beat 20 . Also, the difference between the acceleration and late deceleration rates was computed (Lang \& Hnatiow, 1962).

All measures were compared between Stimuli 1, 2, and 14 with the t test for correlated means.

\section{RESULTS}

Response Shape

The finger pulse response was a monophasic vasoconstriction. The mean maximum constriction occurred at about the seventh poststimulus pulse, and its magnitude was approximately a $45 \%$ decrease in pulse amplitude from the prestimulus level. As the timing of this response was so consistent over Ss, the decrease in pulse amplitude is clearly evident in the pulse-by-pulse average curves. There were no significant differences between FP responses for Stimuli 1, 2, and 14.

The forehead pulse response was biphasic. The initial mean maximum vasoconstriction of $20 \%$ occurred at about the 5th pulse and was followed by maximum vasodilation of $15 \%$ to $28 \%$ at the 9 th to the 13th pulse. As with the finger pulse, the timing of the forehead-pulse constriction was rather constant over Ss and is clearly evident in the puise-by-pulse average curves. In

\section{PULSE BY PULSE} AVERAGE 
Table 1

Correlation of Response with Prestimulus Level and Correlations Between Responses. Pearson Correlation Coefficient, $r$

\begin{tabular}{|c|c|c|c|c|c|c|c|c|c|c|c|c|c|c|c|c|c|c|}
\hline & \multicolumn{2}{|c|}{ Prestimulus } & \multirow{2}{*}{$\frac{\text { Level }}{14}$} & \multicolumn{3}{|c|}{ FP Constriction } & \multicolumn{3}{|c|}{ HP Constriction } & \multicolumn{3}{|c|}{ HP Dilation } & \multicolumn{3}{|c|}{$\begin{array}{c}\text { HR Early } \\
\text { Deceleration }\end{array}$} & \multicolumn{3}{|c|}{ HR Acceleration } \\
\hline & 1 & 2 & & 1 & 2 & 14 & 1 & 2 & 14 & 1 & 2 & 14 & 1 & 2 & 14 & 1 & 2 & 14 \\
\hline $\begin{array}{l}\text { FP Constriction } \\
\text { HP Constriction } \\
\text { HP Dilation } \\
\text { HR Early Deceleration } \\
\text { HR Acceleration } \\
\text { HR Late Deceleration } \\
\text { HR Accel.-Late Decel. }\end{array}$ & $\begin{array}{l}+.72 \\
+.16 \\
+.36 \\
+.61 \\
-.60 \\
+.50 \\
-.27\end{array}$ & $\begin{array}{l}+.74 \\
+.60 \\
+.25 \\
+.22 \\
-.42 \\
+.45 \\
-.01\end{array}$ & $\begin{array}{l}+.84 \\
+.65 \\
-.25 \\
+.34 \\
-.49 \\
-.13 \\
-.49\end{array}$ & $\begin{array}{r}+.32 \\
-.41 \\
-.27 \\
+.32 \\
+.09\end{array}$ & $\begin{array}{r}+.60 \\
-.22 \\
-.03 \\
+.34 \\
-.18\end{array}$ & $\begin{array}{l}+.45 \\
-.31 \\
-.16 \\
-.09 \\
+.38\end{array}$ & $\begin{array}{r}-.26 \\
-.37 \\
+.63 \\
-.30\end{array}$ & $\begin{array}{r}-.34 \\
-.35 \\
+.70 \\
-.37\end{array}$ & $\begin{array}{l}-.02 \\
-.50 \\
+.51 \\
+.09\end{array}$ & $\begin{array}{r}+.03 \\
-.05 \\
+.01\end{array}$ & $\begin{array}{r}-.17 \\
-.41 \\
+.28\end{array}$ & $\begin{array}{r}-.15 \\
+.06 \\
+.07\end{array}$ & $\begin{array}{l}-.42 \\
-.01\end{array}$ & $\begin{array}{r}-.57 \\
+.44\end{array}$ & $\begin{array}{l}-.31 \\
-.26\end{array}$ & -.51 & -.51 & +.16 \\
\hline
\end{tabular}

$r=.40, p<.05 ; r=.60, p<.001$

contrast, the pulse where maximum vasodilation occurred varied over $S s$, thus tending to smear out this phase in the average curves. HP responses did not differ significantly for Stimuli 1, 2, and 14 .

The heart-rate response was more complex but predominantly biphasic. $\mathbf{A}$ mean maximum acceleration of 7 to $15 \mathrm{bpm}$ at about Poststimulus Pulse 4 preceded a mean maximum deceleration of $8 \mathrm{bpm}$ at Pulse 11. Here again, the temporal variability of the deceleratory response tended to obscure it on the pulse-by-pulse average curves; however, the acceleratory response is plainly seen. The mean HR early decelerations (scored between Poststimulus Beats 1-3) were +3.3 , $-2.0,-0.4$ bpm for Stimuli 1,2 , and 14 , respectively. Thus, to the first stimulus, the predominant response in this 1.3 beat period was acceleration. However, to the second stimulus, there was a deceleration from prestimulus level. Stimulus 14 showed a small early deceleration. The early deceleration is apparent in the pulse-by-pulse average curves of Fig. 1 and is depicted in the response diagrams. HR early deceleration increased and HR acceleration decreased significantly $(p<.01)$ between Stimuli 1 and 2 . Neither measure showed significant differences between Stimuli 1 and 14 and Stimuli 2 and 14. HR late deceleration showed no significant difference between the three stimuli tested. The acceleration minus late deceleration score decreased significantly between Stimuli 1 and 2 and between Stimuli 1 and 14, but not between Stimuli 2 and 14.

\section{Response and}

Prestimulus Level

Stimuli 1, 2, and 14 were also used in this analysis. For all three stimuli, the larger the prestimulus FP amplitude the greater the magnitude of the evoked vasoconstriction (see Note 1; Table 1, left column). HP constriction was also similarly significantly correlated with its prestimulus level for Stimuli 2 and 14. The HP vasodilation response was not significantly correlated with the prestimulus amplitude.

Magnitude of HR acceleration was negatively correlated with prestimulus rate, while the mignitudes of early and late deceleration were positively correlated with the prestimulus rate. The higher the prestimulus heart rate, the smaller the acceleration response, and, conversely, the larger the deceleration responses. The acceleration minus late deceleration was not significantly correlated with the prestimulus level.

\section{Interrelationship of Responses}

The cardiac acceleration and the forehead vasoconstriction occurred almost simultaneously, as did cardiac late deceleration and forehead vasodilation. Midway between these two responses, finger vasoconstriction reached its maximum. When it occurred, HR early deceleration preceded all other responses. Results of correlations between responses are presented in Table 1 , right columns. There were significant positive correlations between the magnitude of FP and HP constriction responses for Stimuli 2 and 14, but FP magnitude was not significantly related to any of the HR responses. Magnitude of HP constriction was significantly correlated with HR acceleratory change for Stimuli 1, 2, and 14. HP constriction and HR early deceleration were negatively related. HP dilation did not show a consistent relation to any of the HR scores. The magnitude of the HR acceleratory response was significantly negatively correlated with the magnitude of both HR deceleratory responses (see Note 2). HR early and late decelerations were not consistently related. HP constriction and dilation respenses were not significantly correlated.

\section{DISCUSSION}

Our finding that the finger-pulse response was monophasic vasoconstriction is consistent with previous reports (Burch, 1963; Sokolov, 1963; Uno \& Grings, 1965). Our forehead-pulse response of constriction and then dilation is very similar to that found during waking by Royer (1965) and Raskin et al (1969) and during sleep by Hord \& Ackerland (1968). Sokolov (1963), using a pressure-type plethysmograph to measure volumetric changes of the vasculature in surface area supplied by the temporal artery, reported only vasodilation to moderate auditory stimuli. The difference in results can be attributed to the different site of measurement and especially the method of measurement. We measured pulse amplitude to detect the rather fast changes in arterial tone. On the other hand, Sokolov measured the relatively longer-term changes (dc recording) in blood content of the forehead. A comparison of these two methods of measurement was well demonstrated by Raskin et al (1969). They showed that a decrease in pulse amplitude can occur with an increase in blood content. Fox et al (1960) have shown that the vessels of the forehead are controlled by both sympathetic vasoconstrictor and vasodilator nerves.

The complex HR response to our moderate auditory stimulus was very similar to those recently reported by other investigators using stimuli of various intensities (40-80 dB), frequencies, and rise times (Roessler et al, 1969; Raskin et al, 1969; Smith \& Strawbridge, 1969; Connor \& Lang, 1969). No obvious relation was observed between HR response pattern and respiration changes in our study or by others (Roessler et al, 1969; Connor \& Lang, 1969). The large acceleration that seemed to override any early deceleration in the first stimulus may have been produced by a startle response. Since the Ss were in a no-stimulus condition $10 \mathrm{~min}$ prior to the first stimulus, its onset may have startled some of the Ss and elicited the marked HR acceleration (although the FP and HP responses were not different from those in Stimuli 2 and 14). Stimuli 2 and 14 show the more familiar HR response pattern of decelerationacceleration-deceleration. Each of these components may have a particular psychological and physiological cause and effect. It would also seem that stimulus characteristics as well as the subject set can influence each of these components (Berg \& Graham, 1969).

The magnitude of each response was shown to be related to its prestimulus level. FP and HP constriction and $H R$ acceleration all seem to follow the law of initial values (Wilder, 1962; Hord et al, 1964). The size of the response is 
conversely related to its prestimulus level; for example, if the prestimulus pulse is constricted the magnitude of the evoked response will be decreased. If the prestimulus HR is low, the HR acceleration response will be greater, but the early and late decelerations tend to be smaller.

There appears to be a close relation between the forehead-pulse response and the heart-rate response. HP constriction and HR acceleration occur almost simultaneously and are very positively related in magnitude. Forehead vasoconstriction also is positively related to the FP constriction. These responses may represent a common sympathetic discharge. HR early deceleration and HR late deceleration to a lesser degree are negatively correlated with both HR acceleration and HP constriction. HR early deceleration may be an independent initial response that can be overridden or terminated by the HR acceleratory response as part of the sympathetic discharge. The magnitudes of both components, in turn, appear to be dependent upon the HR prestimulus level.

\section{REFERENCES}

BERG, W. K., \& GRAHAM, F. K. Reproducible effects of stimulus intensity on heart rate curves. Paper presented at Society for Psychophysiological Research, Monterey, Calif., October 1969.

BURCH, G. E. Digital theoplethy smographic study of the orienting reflex in man. Psychosomatic Medicine, 1969, 23, 403-412.

CONNOR, W. H., \& LANG, P. Cortical slow-wave and cardiac rate responses in stimulus orientation and reaction time conditions. Journal of Experimental Psychology, 1969, 82, 310-320.

FOX, R. H., GOLDSMITH, R., \& KIDD, D. J. Cutaneous vasomotor nerves in the human ear and forehead. Journal of Physiology, London, $1960,150,12 P-13 P$.

HORD, D. J., \& ACKERLAND, V.
Non-habituating autonomic systems during sleep. Paper presented at meeting of the Western Psychological Association, San Diego, October 1968.

HORD, D. J., JOHNSON, L. C., \& LUBIN, A. Differential effect of the law of initial value (LIV) on autonomic variables. Psychophysiology, 1964, 1, 79-87.

LANG, P. J., \& HNATIOW, M. Stimulus repetition and the heart rate response. Joumal of Comparative \& Physiological Psychology, 1962, 65, 781-785.

RASKIN, D. C., KOTSES, H., \& BEVER, J. Cephalic vasomotor and heart rate measures of orienting and defensive reflexes. Psychophysiology, 1969, 6, 149-159.

ROESSLER, R., COLLINS, F., \& BURCH, N. R. Heart rate response to sound and light. Psychophysiology, 1969, 5, 359-369.

ROYER, F. L. Cutaneous vasomotor components of the orienting reflex. Behaviour Research \& Therapy, 1965, 3, 161-170.

SMITH, D. B., \& STRAWBRIDGE, P. J. The heart rate response to a brief auditory and visual stimulus. Psychophysiology, 1969, 6, 317-329.

SOKOLOV, E. N. Perception and the conditioned reflex. New York: Macmillan, 1963.

UNO, T., \& GRINGS, W. W. Autonomic components of orienting behavior. Psychophysiology, 1965, 1, 311-321.

WILDER, J. Basimetric approach (law of initial value) to biological rhythms. Annals of New York Academy of Science, 1962, 98, 1211-1220.

\section{NOTES}

1. In computing correlations between pulse-amplitude responses and prestimulus level, the difference between the poststimulus pulse-amplitude response and the first prestimulus pulse amplitude was compared to the average amplitude of the second and third prestimulus pulses, post-pre 1 vs $\left(\right.$ pre $_{2}+$ pre $\left._{3}\right) / 2$. The same method was used with the heart-rate responses. This avoided spurious correlations due to common error of measurement.

2. To avoid spurious correlations due to common error of measurement, new HR deceleration scores and HP dilation scores were computed using different prestimulus scores-HR accel.post - pre 1 vs HR decel.post - pre $_{2} ; \mathrm{HP}$ (constr.post - pre ) $^{\text {pre }}{ }_{1}$ ys HP (dilat.post - pre $_{2}$ )/pre pre $_{2}$. or frequency of concept solution was found in nonmediated learning.

In a series of studies relating intelligence to concept learning, Osler and associates found that: (1) Ss of superior intelligence solve concepts more rapidly than lower-intelligence Ss; and (2) high-intelligence Ss learn insightfully, whereas lower-intelligence $S s$ learn by associative incremental learning (Osler \& Fivel, 1961; Osler \& Trautman, 1961; Osler \& Weiss, 1962). In contrast, recent studies (Hamilton \& Saltz, 1969; Saltz \& Hamilton, 1969) have found that: (1) when precriterion errors for concept solvers are Vincentized into quartiles no evidence of precriterion improvement is found for Ss of high or lower intelligence, and (2) intelligence is related to the frequency with which Ss solve problems but not to the speed of solution among the solvers. The Ss in these studies were all children.

In a previous study, Jacobson, Millham, \& Berger (1969) found that Ss high in intelligence demonstrated more rapid concept learning than $S$ s lower in intelligence in a mediation condition but not in a nonmediation condition. These results were taken as supporting the position of Osler and associates. However, Jacobson, Millham, and Berger did not divide Ss into concept solvers and nonsolvers as recommended by Saltz and Hamilton.

The present study further tested the hypothesis of Saltz and Hamilton that intelligence is related to frequency but not to speed of concept solution by reevaluating the data of Jacobson, Millham, and Berger. In the present analysis, Ss were divided into high-, medium-, and low-intelligence groups; Ss were divided also into concept solvers or nonsolvers in mediated learning and into solvers or nonsolvers in nonmediated learning. The effects of intelligence were then related to the frequency and speed of concept solution.

\section{METHOD}

The Ss were 115 undergraduate volunteers. Each $S$ learned one of two paired-associate lists and a concept list (Mednick \& Freedman, 1960). The first paired-associate list (PAL-1) was designed to facilitate the attainment of the concept "white" in subsequent concept learning. On this list, the learning of 4 of the 12 word pairs facilitated the attainment of "white" in later concept learning. The remaining word pairs on this list were neutral with regard to "white." The second paired-associate list (PAL-2) was similarly designed but facilitated the attainment of and frequency of problem solution in concept learning was evaluated. Differences in intelligence were found to predict the frequency but not the speed of concept solution in mediated learning. No relationship between intelligence and speed
The relationship of intelligence to speed

LEONARD I. JACOBSON 33124

Psychon. Sci., 1970, Vol. 19 (6) 